\title{
Erratum to: Infinitely many solutions for resonant cooperative elliptic systems with sublinear or superlinear terms
}

\author{
Guanwei Chen · Shiwang Ma
}

Published online: 19 November 2013

(C) Springer-Verlag Berlin Heidelberg 2013

\section{Erratum to: Calc Var DOI 10.1007/s00526-012-0581-5}

We mention that there are two errors in the original article.

\section{The proof of Lemma 2.4}

There is an error in the proof of Lemma 2.4 of the original article. In fact, the inequality

$$
|U| \leq \varepsilon<R_{2}, \quad \forall 0<\varepsilon<R_{2} \quad\left(R_{2}\right. \text { is a constant) }
$$

in the proof of Lemma 2.4 is incorrect $\left(R_{2}>0\right.$ is a constant). Indeed, if $(*)$ holds, then $U=0$. But we need $U \neq 0$ in Lemma 2.4. Therefore, the proof of Lemma 2.4 fails.

However, we can still complete the proof of Lemma 2.4 by making some changes in the original condition $\left(A F_{2}\right)$. We should mention that the changes will not influence the remainder framework of the original article. For the readers convenience, we state the previous conditions $\left(A F_{1}\right)-\left(A F_{3}\right)$ in the original article as follows:

$\left(\mathbf{A F}_{1}\right) F(x, U) \geq 0, \forall(x, U) \in \Omega \times \mathbb{R}^{2}$, and there exist constants $\mu \in[1,2)$ and $R_{1}>0$ such that

$$
(\nabla F(x, U), U) \leq \mu F(x, U), \quad \forall x \in \Omega \text { and }|U| \geq R_{1} .
$$

The online version of the original article can be found under doi:10.1007/s00526-012-0581-5.

\section{G. Chen $(\varangle)$}

School of Mathematics and Statistics, Anyang Normal University, Anyang 455000, Henan Province, People's Republic of China e-mail: guanweic@163.com

S. Ma

School of Mathematical Sciences and LPMC, Nankai University, Tianjin 300071, People's Republic of China e-mail: shiwangm@163.net 
$\left(\mathbf{A F}_{2}\right) \lim _{|U| \rightarrow 0} \frac{F(x, U)}{|U|^{2}}=\infty$ uniformly for $x \in \Omega$, and there exist constants $c_{2}, R_{2}>0$ such that

$$
F(x, U) \leq c_{2}|U|, \quad \forall x \in \Omega \text { and }|U| \leq R_{2} .
$$

$\left(\mathbf{A F}_{3}\right) \liminf \operatorname{sit\rightarrow \infty }_{|U|} \frac{F(x, U)}{|U|} \geq d>0$ uniformly for $x \in \Omega$.

In order to give a correct proof of Lemma 2.4, we change the condition $\left(A F_{2}\right)$ to $\left(\mathbf{A F}_{\mathbf{2}}^{\prime}\right) \quad$ There exist constants $a \in[1,2)$ and $c_{2}, c_{2}^{\prime}, R_{2}>0$ such that

$$
F(x, U) \geq c_{2}^{\prime}|U|^{a}, \quad \forall x \in \Omega \text { and } U \in \mathbb{R}^{2}
$$

and

$$
F(x, U) \leq c_{2}|U|, \quad \forall x \in \Omega \text { and }|U| \leq R_{2} .
$$

Remark Obviously, $\left(A F_{2}^{\prime}\right)$ - $(i)$ implies that $\left(A F_{3}\right)$ and $F(x, U) \geq 0$ for all $(x, U) \in \Omega \times \mathbb{R}^{2}$ hold. Therefore, the conditions $\left(A F_{3}\right)$ and $F(x, U) \geq 0, \forall(x, U) \in \Omega \times \mathbb{R}^{2}$ in $\left(A F_{1}\right)$ can be omitted.

Next, we give the correct proof of Lemma 2.4.

Lemma 2.4 (the original article) Assume that $\left(A F_{1}\right)$ and $\left(A F_{2}^{\prime}\right)$ hold. Then there exist a positive integer $k_{1}$ and two sequences $0<r_{k}<\rho_{k} \rightarrow 0$ as $k \rightarrow \infty$ such that

$$
\begin{gathered}
\alpha_{k}(\lambda):=\inf _{U \in Z_{k},\|U\|=\rho_{k}} \Phi_{\lambda}(U)>0, \quad \forall k \geq k_{1}, \\
\xi_{k}(\lambda):=\inf _{U \in Z_{k},\|U\| \leq \rho_{k}} \Phi_{\lambda}(U) \rightarrow 0 \text { as } k \rightarrow \infty \text { uniformly for } \lambda \in[1,2]
\end{gathered}
$$

and

$$
\beta_{k}(\lambda):=\max _{U \in Y_{k},\|U\|=r_{k}} \Phi_{\lambda}(U)<0, \quad \forall k \in \mathbb{N}
$$

where $Y_{k}=\bigoplus_{m=1}^{k} X_{m}$ and $Z_{k}=\overline{\bigoplus_{m=k}^{\infty} X_{m}}$ for all $k \in \mathbb{N}$.

Proof (a) Firstly, we show that (1.1) and (1.2) hold. Obviously, $Z_{k} \subset W^{+}$for all $k \geq k_{1}:=$ $l_{0}+1$ (see Sect. 2.2 in the original article). It is not hard to check that $\left(A F_{1}\right),\left(A F_{2}^{\prime}\right)$ and the definition of $\widetilde{F}$ imply that

$$
\widetilde{F}(x, U) \leq a_{1}\left(|U|+|U|^{\mu}\right), \quad \forall x \in \Omega \text { and } U \in \mathbb{R}^{2}
$$

for some constant $a_{1}>0$. It follows from the definition of $\Phi_{\lambda}$ that for any $k \geq k_{1}$ and $U \in Z_{k}$ there holds

$$
\Phi_{\lambda}(U) \geq \frac{1}{2}\|U\|^{2}-2 \int_{\Omega} \widetilde{F}(x, U) d x \geq \frac{1}{2}\|U\|^{2}-2 a_{1}|U|_{1}-2 a_{1}|U|_{\mu}^{\mu}, \quad \forall \lambda \in[1,2],
$$

where $|\cdot|_{p}$ denotes the norm of $L^{p}(\Omega) \times L^{p}(\Omega)$. Let

$$
l_{k}(1):=\sup _{U \in Z_{k} \backslash\{0\}} \frac{|U|_{1}}{\|U\|}, \quad l_{k}(\mu):=\sup _{U \in Z_{k} \backslash\{0\}} \frac{|U|_{\mu}}{\|U\|}, \quad \forall k \in \mathbb{N} .
$$


Then

$$
l_{k}(1) \rightarrow 0 \text { and } l_{k}(\mu) \rightarrow 0 \text { as } k \rightarrow \infty
$$

by the Rellich embedding theorem (see [4]). Hence, for any $k \geq k_{1}$ and $U \in Z_{k}$, (1.4) and (1.5) imply

$$
\Phi_{\lambda}(U) \geq \frac{1}{2}\|U\|^{2}-2 a_{1} l_{k}(1)\|U\|-2 a_{1} l_{k}^{\mu}(\mu)\|U\|^{\mu}, \quad \forall \lambda \in[1,2] .
$$

Let

$$
\rho_{k}:=16 a_{1} l_{k}(1)+\left(16 a_{1} l_{k}^{\mu}(\mu)\right)^{1 /(2-\mu)}, \quad k \in \mathbb{N} .
$$

Then by (1.6) and $\mu<2$, we have

$$
\rho_{k} \rightarrow 0 \text { as } k \rightarrow \infty \text {. }
$$

For any $k \geq k_{1},(1.7)$ together with (1.8) implies that

$$
\alpha_{k}(\lambda):=\inf _{U \in Z_{k},\|U\|=\rho_{k}} \Phi_{\lambda}(U) \geq \rho_{k}^{2} / 2-\rho_{k}^{2} / 8-\rho_{k}^{2} / 8=\rho_{k}^{2} / 4>0 .
$$

That is, (1.1) holds.

For any $k \geq k_{1}$ and $U \in Z_{k}$ with $\|U\| \leq \rho_{k}$, (1.7) implies that $\Phi_{\lambda}(U) \geq-2 a_{1} l_{k}(1) \rho_{k}$ $-2 a_{1} l_{k}^{\mu}(\mu) \rho_{k}^{\bar{\mu}}$. Observing that $\Phi_{\lambda}(0)=0$ by $\left(A F_{2}^{\prime}\right)$ and the definition of $\widetilde{F}$, thus

$$
0 \geq \inf _{U \in Z_{k},\|U\| \leq \rho_{k}} \Phi_{\lambda}(U) \geq-2 a_{1} l_{k}(1) \rho_{k}-2 a_{1} l_{k}^{\mu}(\mu) \rho_{k}^{\mu}, \quad \forall k \geq k_{1} .
$$

It follows from (1.6) and (1.9) that

$$
\xi_{k}(\lambda):=\inf _{U \in Z_{k},\|U\| \leq \rho_{k}} \Phi_{\lambda}(U) \rightarrow 0 \text { as } k \rightarrow \infty \text { uniformly for } \lambda \in[1,2] .
$$

That is, (1.2) holds.

(b) Now, we show that (1.3) holds. Since norms $|\cdot|_{a}$ and $\|\cdot\|$ are equivalent on finitedimensional space $Y_{k}$, there exists a constant $C_{k}>0$ such that

$$
|U|_{a} \geq C_{k}\|U\|, \quad \forall U \in Y_{k}, \quad \forall k \in \mathbb{N},
$$

where $a \in[1,2)$ is given in $\left(A F_{2}^{\prime}\right)$. Thus, by $(1.10),\left(A F_{2}^{\prime}\right)$ and the definition of $\widetilde{F}$, for any $k \in \mathbb{N}$ and $U \in Y_{k}$, we have

$$
\begin{aligned}
\Phi_{\lambda}(U) \leq \frac{1}{2}\left\|U^{+}\right\|^{2}-\int_{\Omega} \tilde{F}(x, U) d x & \leq \frac{1}{2}\|U\|^{2}-c_{2}^{\prime}|U|_{a}^{a} \\
& \leq \frac{1}{2}\|U\|^{2}-c_{2}^{\prime} C_{k}^{a}\|U\|^{a}, \quad \forall \lambda \in[1,2] .
\end{aligned}
$$

If we choose

$$
0<r_{k}<\min \left\{\rho_{k},\left(c_{2}^{\prime} C_{k}^{a}\right)^{1 /(2-a)}\right\}, \quad \forall k \in \mathbb{N},
$$

then (1.11) implies

$$
\beta_{k}(\lambda):=\max _{U \in Y_{k},\|U\|=r_{k}} \Phi_{\lambda}(U) \leq-r_{k}^{2} / 2<0, \quad \forall k \in \mathbb{N} .
$$

That is, (1.3) holds. Therefore, the proof is finished. 


\section{The proof of Theorem 1.1}

In the proof of Theorem 1.1 in the original article, the authors only proved the following result:

Claim $1\left\{U_{j}\right\}$ is bounded in $W$.

Note that the proof of Theorem 1.1 is based on Lemma 2.1 in the original article, thus we must get the following result:

Claim $1^{\prime}\left\{U_{j}\right\}$ possesses a strongly convergent subsequence in $W=W^{0} \oplus W^{-} \oplus W^{+}$, where $\left\{U_{j}\right\} \subset Y_{j}$ and $Y_{j}$ is a finite dimensional subspace $(j \in \mathbb{N})$.

Since $j \in \mathbb{N}$, Claim 1 is not enough to get the result of Claim $1^{\prime}$. Therefore, to complete the proof of Theorem 1.1, we not only need to prove Claim 1 but also need to prove Claim $1^{\prime}$.

Proof of Claim $1^{\prime}$ By Claim 1 and the fact $\operatorname{dim}\left(W^{0} \oplus W^{-}\right)<\infty$, without loss of generality, we may assume

$$
U_{j}^{-} \rightarrow U^{-}, \quad U_{j}^{0} \rightarrow U^{0}, \quad U_{j}^{+} \rightarrow U^{+} \quad \text { and } \quad U_{j} \rightarrow U \quad \text { as } j \rightarrow \infty
$$

for some $U=U^{0}+U^{-}+U^{+} \in W=W^{0} \oplus W^{-} \oplus W^{+}$. By virtue of the Riesz Representation Theorem, $\left.\Phi_{\lambda_{j}}^{\prime}\right|_{Y_{j}}: Y_{j} \rightarrow Y_{j}^{*}$ and $I^{\prime}: W \rightarrow W^{*}$ can be viewed as $\left.\Phi_{\lambda_{j}}^{\prime}\right|_{Y_{j}}: Y_{j} \rightarrow Y_{j}$ and $I^{\prime}: W \rightarrow W$ respectively, where $Y_{j}^{*}$ and $W^{*}$ are the dual spaces of $Y_{j}$ and $W$, respectively. Note that (2.23) in the original article implies

$$
0=\left.\Phi_{\lambda_{j}}^{\prime}\right|_{Y_{j}}=U_{j}^{+}-\lambda_{j}\left[U_{j}^{-}+\chi_{j} I^{\prime}\left(U_{j}\right)\right], \quad \forall j \in \mathbb{N},
$$

where $\chi_{j}: W \rightarrow Y_{j}$ is the orthogonal projection for all $j \in \mathbb{N}$, that is,

$$
U_{j}^{+}=\lambda_{j}\left[U_{j}^{-}+\chi_{j} I^{\prime}\left(U_{j}\right)\right], \quad \forall j \in \mathbb{N} .
$$

Under the definition of $\widetilde{F}$ and the assumptions of $F$, by the standard argument (see $[1,3]$ ), we know $I^{\prime}: \quad W \rightarrow W^{*}$ is compact. Therefore, $I^{\prime}: \quad W \rightarrow W$ is also compact. By the compactness of $I^{\prime}$ and (2.1), we get the right-hand side of (2.2) converges strongly in $W$ and hence $U_{j}^{+} \rightarrow U^{+}$in $W$. Combining this with (2.1), we have $U_{j} \rightarrow U$ in $W$. Therefore, Claim $1^{\prime}$ is true.

Now from the last assertion of Lemma 2.1, we know that $\Phi=\Phi_{1}$ has infinitely many nontrivial critical points. Therefore, problem (1.1) in the original article possesses infinitely many nontrivial solutions. That is, Theorem 1.1 in the original article is true.

\section{References}

1. Benci, V., Rabinowitz, P.H.: Critical point theorems for indefinite functionals. Invent. Math. 52, 241-273 (1979)

2. Chen, G., Ma, S.: Infinitely many solutions for resonant cooperative elliptic systems with sublinear or superlinear terms. Calc. Var. doi:10.1007/s00526-012-0581-5

3. Rabinowitz, P.H.: Minimax methods in critical point theory with applications to differential equations, CBMS Reg. Conf. Ser. Math., vol. 65. American Mathematical Society, Providence (1986)

4. Willem, M.: Minimax Theorems. Birkhäuser, Boston (1996) 